\title{
Phonological factors in lexical access: Evidence from an auditory lexical decision task
}

\author{
WILLIAM MILBERG \\ Veterans Administration Medical Center, Boston, Massachusetts \\ and GRECC VA Medical Center, West Roxbury, Massachusetts \\ SHEILA BLUMSTEIN \\ Veterans Administration Medical Center, Boston, Massachusetts \\ and Brown University, Providence, Rhode Island \\ and \\ BARBARA DWORETZKY \\ Veterans Administration Medical Center, Boston, Massachusetts
}

\begin{abstract}
A group of subjects performed a lexical decision task in which target words were preceded by a number of different prime types: semantically related words, nonwords in which the initial phoneme of the semantically related word was distorted by one phonetic feature, nonwords in which the initial phoneme of the related word was distorted by more than one phonetic feature, and unrelated words. The results showed a monotonic relationship between phonetic distortion and lexical decision facilitation. Lexical access appears to take into account possible noise or distortion of the speech signal, so that a nonword stimulus that is phonetically related to an actual lexical entry is, in some sense, "normalized" and processed as an actual lexical entry.
\end{abstract}

Although it is usually assumed that the mental lexicon contains information about the acoustic or phonological characteristics of its constituent words, it is unclear how much of this information is used to interpret an incoming speech signal. There has been considerable disagreement over the degree to which processes involved in the lexical interpretation of speech are dependent on the speech signal itself.

Some models of speech perception assume that words are stored as sequences of phonemes or morphemes that must be matched exactly before recognition can occur. In the absence of an exact match, the user may use context or other "top-down" sources of information to interpret the speech stimulus. For example, Marslen-Wilson and Welch (1978) argued that speech recognition begins with the first sound of the word the subject hears. Lexical processing is then limited to the subset or cohort of words that begins with that sound. As additional sounds of the word are heard, the cohort is further narrowed until a single word is uniquely specified. This model is dependent on the listener's accurate reception of the acoustic or phonemic content of the speech signal, particularly for the initial sounds of the word or utterance. If the initial phonemes are incorrectly interpreted, the listener is forced to search through an irrelevant cohort of words.

Another possibility is that the lexicon stores basic information about the acoustic or phonetic characteristics of the speech signal (McClelland \& Elman, 1986; Stevens \& Blumstein, 1981) without requiring an exact match for lexical ac-

This research was supported by NIH Grant NS 22282 to Sheila Blumstein at Brown University, NIH Grant NS 06209 to Harold Goodglass at Boston University School of Medicine, and by VA Merit Review 09744-3765-001 and NIA Grant RO 1 AG 03354-03 to William Milberg. Address correspondence to William Milberg, Geriatric Research, Education and Clinical Center, 1400 VFW Parkway, Veterans Administration Medical Center, West Roxbury, MA 02132. cess to proceed. In particular, the lexical access system could allow for the acoustic signal to be "normalized" or corrected. In the absence of an exact match, the listener would still be able to consider the set of vocabulary items partially determined by the phonetic content of the signal. The listener could still use context in the ultimate interpretation of a word, but processing would not fail when the initial parts of the word were incorrectly heard.

A final possibility is that lexical access is so underdetermined by the speech signal that it must always proceed through top-down routines for lexical interpretation. In this case, wide variations in the acoustic signal would be tolerated and many irrelevant lexical candidates would be considered. The final interpretation of a word would depend on the direct application of the listener's world knowledge. Models that place a great deal of weight on these top-down processes have been proposed (e.g., Forster, 1978), although all seem to set some limits to lexical access in the acousticphonetic structure of the word.

In the current study, a lexical decision paradigm was used to examine the extent to which lexical access can occur in the face of a phonologically distorted speech signal. Results of lexical decision studies have shown that lexical decisions are made more quickly when target words are preceded by a word of related meaning than when preceded by words that are unrelated (Antos, 1979; Meyer \& Schvaneveldt, 1977; Posner \& Snyder, 1975; Radeau, 1983). This "semantic facilitation" effect occurs even if the likelihood of predicting the identity of the target is minimized by lowering the proportion of related word pairs (Tweedy, Lapinski, \& Schvaneveldt, 1977), by using extremely short primetarget interstimulus intervals (Neely, 1977), or by obscuring the identity of the prime with a pattern mask (Fowler, Wolford, Slade, \& Tassinary, 1981; Marcel, 1980). Hence, 
this paradigm can be used to study lexical access processes that are not dependent on the top-down analysis of the word's semantic characteristics.

In this study, the initial phoneme of the prime was changed by one or more phonetic features, keeping the succeeding rhyming part of the prime word constant. This change altered the lexical status of the prime from a real word to a nonword (e.g., cat to gat or wat). In this manner, the sensitivity of the semantic facilitation effect to the explicit alteration of a speech-related acoustic variable could be examined. Target words served as their own controls across conditions. These conditions included an unchanged prime related to the target (e.g., cat-dog), a phonetically altered prime with the initial consonant one phonetic feature from the prime (e.g., gat-dog), another phonetically altered prime with the initial consonant more than one phonetic feature from the prime (e.g., wat-dog), and a real word semantically unrelated to the target (e.g., table-dog) to serve as a baseline for the other conditions.

The relationship between acoustic/phonetic distance and response latency may take at least three broad forms. The hypothetical curves representing these three relationships are shown in Figure 1. The ordinate of each curve represents the response latency of the lexical decision made for a realword target following a prime word. The abscissa represents the four priming conditions in the proposed experiment. Included are the three levels of phonetic distance and a baseline consisting of an unrelated word. It is assumed that these four conditions form a roughly ordinal scale of "relatedness" to the target. In the case of Curve A (phonologically underdetermined), all three levels of phonetic distance facilitate decision latencies equally, relative to the unrelated word baseline; the initial consonant is irrelevant, and only rhyming (shared vowel plus consonant) is sufficient to influence lexical decision time. In the case of Curve B (phonetic feature determined), there is an incremental change as a function of phonetic distance relative to the unrelated word base-

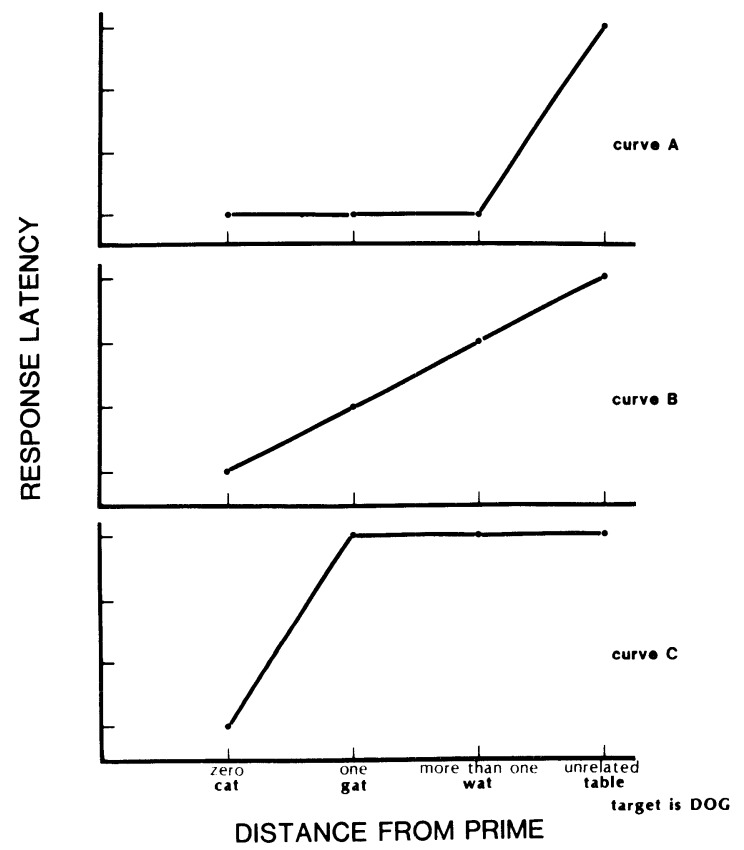

Figure 1. Possible relationships between lexical decision latencies and phonological distortion. line. Each change in the number of shared phonetic features (resulting in nonword primes similar to a semantically related prime) affects reaction time. This sort of relationship would suggest that lexical access is built on a phonetic feature system that is similar in format to the system responsible for speech perception (Stevens \& Blumstein, 1981).

Finally, in Curve C (phonologically overdetermined), semantic facilitation is observed relative to the unrelated word baseline if and only if a phonologically accurate semantically related real word is presented. No facilitation will occur relative to the unrelated word baseline even for phonologically related nonwords. For this system, the input must match the stored phonological representation of a word in the lexicon, and this lexicon would not be addressable by nonwords. This result would be predicted by "cohort models" of lexical access (Marslen-Wilson \& Welch, 1978), and suggests that lexical access is determined by specific central phonological representations, without necessarily being sensitive to phonetic features used in the auditory perception of speech.

\section{METHOD}

\section{Subjects}

The subjects in this experiment were 12 undergraduates attending Brown University. They were paid $\$ 3$ for participating in one half-hour session. The subjects were assigned sequentially to one of two test tapes that differed in the order of stimulus presentation.

\section{Stimuli}

The stimuli used in this study consisted of high-frequency pairs of words and nonwords, with the first member of the pair considered the prime and the second member considered the target. Six types of these prime-target pairs were constructed in order to create four priming conditions for the real words ("yes" responses) and two priming conditions for the nonwords ("no" responses). The first four conditions contained the same 15 real-word monosyllabic targets preceded either by a related (semantically associated) word (determined by the word association norms of Bousfield, Cohen, Whitmarsh, \& Kincaid, 1961) (e.g., cat-dog), a nonword differing from the semantically related prime by one distinctive feature (either voicing or place) in the initial phoneme position (e.g., gat-dog), a nonword differing from the semantically related prime by more than one distinctive feature in the initial phoneme position (e.g., wat-dog), or a real word semantically unrelated (neutral) with respect to the target word (e.g., table-dog). The last two types of pairs (totaling 45) consisted of nonword targets preceded by either a real word (15 in number) (e.g., cat-jand) or a nonword prime (30 in number) (e.g., wat-naib). The nonwords were all pronounceable English sequences differing from real words by one phoneme (e.g., flower-flowem, circle-pircle).

In summary, the test stimuli consisted of 105 trials: 15 real-word targets were preceded by 15 semantically related words, 15 nonwords derived by changing one distinctive feature of the initial phoneme position of the semantically related word, 15 nonwords derived by changing more than one distinctive feature in the initial phoneme position of the semantically related words, 15 semantically unrelated real words, and 45 nonword targets ( 15 preceded by real-word primes and 30 preceded by nonword primes).

The stimulus pairs were presented in four blocks, each of which contained the 15 real-word targets ("yes" responses) and 11 different nonword targets ("no"' responses). Based on a previous pilot study, the order of presentation of stimulus pairs was shown to have an effect on response time. To control for order effects, two different test tapes were constructed in which the order of the stimulus presentation was reversed. Similar targets were separated within a list as much as possible, and conditions were presented pseudorandomly within the list (using all possible orders of prime words such that half of the phonologically derived primes appeared before their related real-word counterparts and half of them appeared after their related real-word counterparts). All possi- 
ble orders of types of pairs were represented across the two tapes to control for order effects and to minimize repetition effects (Scarborough, Cortese, \& Scarborough, 1977). Order was included as a factor in the initial analysis of the data. There was an 8-sec interval of silence between each stimulus pair and a .5-sec interval of silence between each member within the pair.

Following the primary lexical decision test, the subjects heard the same items from the test, randomly presented one at a time with a 6-sec interval of silence between items, and were again asked to make lexical decisions for these items. Thirty of these items were real words and were presented to determine whether all of the test stimuli and, in particular, the phonologically derived nonword primes were in fact perceived correctly as either words or nonwords. The subjects were provided with five practice trials before beginning the test. These trials contained pairs of unrelated items, none of which appeared in the testing session.

\section{Apparatus}

The apparatus for the experiment consisted of a Crown stereo tape recorder, a Pioneer SA 500A stereo amplifier, a Grason-Stadler voiceoperated relay, a Gerbrands millisecond timer, two pairs of headphones, and a subject response board. The response board consisted of two keys marked "YES" (real word) and "NO" (nonword). The output of the tape recorder was amplified with the stereo amplifier. The output of the amplifier was split so that the channel containing the tone activated the voice-operated relay. With the onset of the target word in each trial, the tone activated the voice-operated relay, which in turn activated the millisecond timer. The subjects heard the stimuli from both channels through sealed headphones. The tape was stopped by pressing one of the two $2 \times 4$ in. marked keys.

\section{Procedure}

The subjects were run individually during one session lasting approximately $30 \mathrm{~min}$. The session was made up of three parts: the five practice trials, the actual test of 105 prime-target pairs, and the individual presentation of the prime stimuli from the main lexical decision experiment. The subject was instructed that for Parts 1 and 2, he/she would hear pairs of sounds. Some of these sounds would be real English words and some of them would be nonsense words. The subject's task was to decide whether or not the second stimulus of each pair was a real word. If it was, they were to press the YES key, and if it was not, the NO key. Responses were made with the subject's preferred hand, which was allowed to rest between the keys after each trial. The subject was told to disregard the first item in the pair, as it was irrelevant to the task. He/she was encouraged to respond as quickly as possible. In Part 3, the subject was required to make lexical decisions about the singly presented prime stimuli.

\section{RESULTS}

Because there was a possibility that a feature effect might result from confusion errors (i.e., misperceiving a nonword prime as the real word from which it was derived), immediately following the lexical decision task, the subjects were asked to classify the auditorily presented primes as words or nonwords. These results were examined as a function of distance from the unaltered prime. Results showed that for two of the words, $58 \%$ of the subjects confused the onefeature-altered prime with the real word (i.e., they heard flower for the rhyming nonword slower and fruit for the rhyming nonword vruit). As a result, the data for all conditions relating to flower and fruit were eliminated from the analysis in the lexical decision study. The remaining 13 target words showed a mean error rate of $2 \%$ in the undistorted prime condition, $0 \%$ in the one-feature-altered condition, and $5 \%$ in the two-feature-altered condition. A one-way analysis of variance (ANOVA) showed no differences between these conditions $[F(2,36)=2.31]$. These results suggest that the subjects perceived the nonword primes as nonwords and not as phonologically related real words. As a result, whatever pat- tern emerged in the lexical decision experiment could not be attributed to patterns of misperception of the prime.

The results of the lexical decision task are shown in Figure 2. Mean lexical decision latencies of correct reponses to the target in the four priming conditions are shown. An ANOVA was conducted to examine the effect of phonetic distance of the initial consonant of the prime on the lexical decision latencies for the target word. In addition, the effect of the position of the semantically related unaltered prime in relation to the two corresponding phonetically altered prime words was included as a separate factor. This was felt to be necessary because, as stated earlier, hearing the unaltered primes before the altered versions of the prime might affect a subject's ability to predict the identity of the target relative to when the unaltered prime appeared in the last condition. The effect of position was not significant $[F(1,11)$ $=1.333$, n.s.], and the position $\times$ phonetic distance interaction was also not significant $[F(3,33)=1.604$, n.s. $]$. The effect of the phonetic distance of the initial phoneme, however, was significant $[F(3,33)=11.4332, p<.01]$, with the shortest lexical decision latencies occurring with the unaltered target, followed in increasing order by the onefeature-altered condition, the two-feature-altered condition, and the unrelated condition.

A Neuman-Keuls test on the differences between adjacent and nonadjacent means revealed that the unaltered condition did not differ from the one-feature-altered condition, but did differ significantly from the two-feature-altered and unrelated conditions. The one-feature-altered condition did not differ from the two-feature-altered condition, but did differ significantly from the unrelated word condition. It appears, therefore, that despite the fact that different phonetic features were changed and combined in each condition, this manipulation had a roughly linear effect on target lexical decision latencies. Indeed, a post hoc trend analysis indicated that a linear trend in the data was significant $[F(1,11)=$ $25.82, p<.01$ ], accounting for $85 \%$ of the total variance. Higher level trends did not approach significance $(F<1)$.

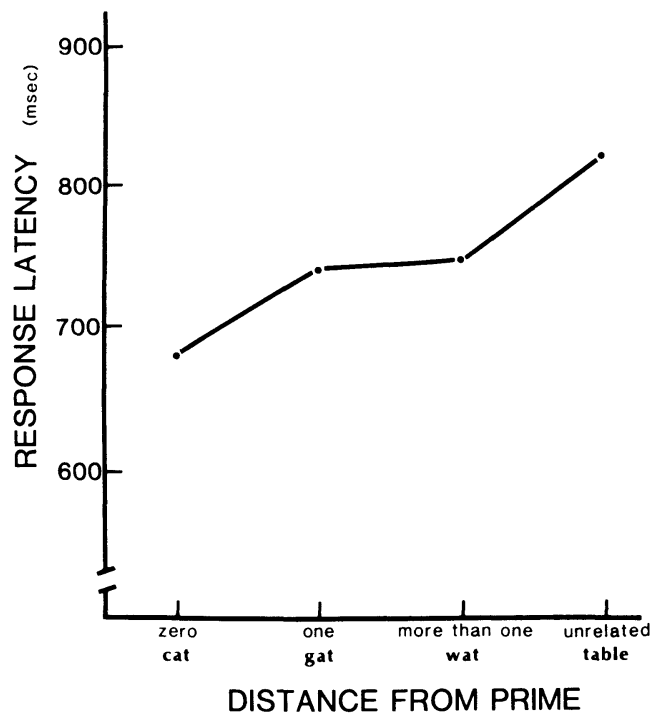

Figure 2. Lexical decision latencies as a function of phonological distance of nonword primes from semantically related primes and unrelated word baseline. 
This analysis suggests that the relationship of phonological distortion to lexical decision reaction time is, if not truly linear, at least a monotonically increasing function with the stimuli used in this study.

These results indicate that semantic facilitation does occur when a prime is presented that is phonetically similar to an actual lexical entry and semantically related to the target word. Nevertheless, the size of the effect is smaller for the phonetically altered primes than for the semantically related real-word prime. This outcome bears a strong resemblance to theoretical Curve B shown in Figure 1.

Higher order (nonlinear) trends would have to have been significant for the results to resemble Curves A or C. In addition, in order for the results to have been comparable to Curve A, both phonetic distance conditions would have had to produce significant priming relative to baseline, and would not have differed from the semantically related prime condition. For the results to have been comparable to Curve C, none of the phonetic distance conditions could have differed from the baseline condition.

\section{DISCUSSION}

The results of the lexical decision experiment showed a monotonic (and perhaps linear) relationship between phonetic distortion and facilitation, with nonword primes facilitating lexical decision latencies as a function of their phonetic relationship to the word semantically related to the target. Unlike the relationship depicted in Curve C of Figure 1, lexical access seems to tolerate variations in the speech signal. However, unlike the situation depicted in Curve A, these variations must be phonologically related to the actual lexical entry. Thus the current results do not support models that suggest that lexical access is critically dependent on reception of the initial sounds of a word (Marslen-Wilson \& Welch, 1978). These models predict that a nonword (even if it shares the final vowel-consonant sounds with a related word) should not access the lexical entry of an actual real word. In addition, an incorrect interpretation of the initial consonant would cause the subject to look up a potential lexical entry in the wrong place in the mental "dictionary."

Lexical access appears to take into account possible noise or distortion of the signal, so that a stimulus that is phonetically related to an actual lexical entry is, in some sense, normalized and treated lexically. This process seems to occur rapidly and, as evidenced by the subjects' ability to discriminate the lexical status of the prime words themselves, does not seem to depend either on misperception or on conscious categorization.

How does the suggested normalization of the speech signal take place? One possibility is that the mechanism responsible for lexical access may try to match any input to actual lexical entries by iteratively searching for entries that are phonetically similar to the input. As a result, the greater the phonetic distance between the nonword and the actual lexical entry, the larger the potential set of words that would have to be searched before a match could be made to the lexical entry. Such a mechanism might result in a phonetic feature effect. This sort of serial search mechanism would still need additional information to disambiguate the word from the set of possible words so that a decision as to final lexical status could be made and would be rather time-consuming to implement. Still, it is quite possible that lexical search occurs rapidly enough to produce a linear relationship between phonetic distance and lexical decision time, and this model could easily be adapted to the assumptions of the "cohort model"' (Marslen-Wilson \& Welch, 1978).

Another possibility is that lexical access uses a feature-counting mechanism with "activation" of a lexical entry varying as a function of the number of shared features or acoustic similarity between the input and the entry. This entry will activate semantically related entries, as has been shown in semantic facilitation studies. The greater the number of shared features or the acoustic similarity between the input and the entry, the greater the degree of activation of semantically related entries without necessarily bringing to threshold the phonologically related entry. For example, gat may prime $\mathrm{dog}$ without first being lexically categorized as cat. Hence, given this threshold effect, lexical access could work in an all-or-none manner and without the subject's awareness. This is a basic assumption of the "logogen model" (Morton, 1969) and is consistent with distinctive-feature-based theories of speech perception (Stevens \& Blumstein, 1981).

A third possibility is that the nonword input is first normalized by a process in which phonetic features related to the input are activated. Thus, whenever a nonword is heard, words that have shared phonetic features are also activated, and the amount of spreading activation to semantically related entries is equivalent. When the subject hears gat, the lexical entry cat (which differs only in the voicing of the initial consonant) is activated, but later than if the input had been cat.

Although the current data do not permit one to choose between these models, they do suggest that a spreading activation framework at the phonetic level can be used to better understand the processes underlying lexical access.

\section{REFERENCES}

ANtos, S. J. (1979). Processing facilitation in a lexical decision task. Journal of Experimental Psychology: Human Perception \& Performance, 5, 527-545.

Bousfield, W. A., Cohen, B. H., Whitmarsh, G. A., \& Kincaid, W. D. (1961). The Connecticut free associational norms (Tech. Rep. No. 35). Storrs, CT: University of Connecticut.

FORSTER, K. I. (1978). Accessing the mental lexicon. In R. J. Wales \& E. C. T. Walker (Eds.), New approaches to language mechanisms. (pp. 257-288). Amsterdam: North-Holland.

Fowler, C. A., Wolford, G., Slade, R., \& TASSinary, L. (1981). Lexical access with and without awareness. Journal of Experimental Psychology: General, 110, 341-362.

MARCEL, A. J. (1980). Conscious and preconscious recognition of polysemous words: Locating the selective effects of prior verbal context. In R. Nickerson (Ed.), Attention and performance VIII (pp. 435-457). Hillsdale, NJ: Erlbaum.

Marslen-Wilson, W. D., \& Welch, A. (1978). Processing interactions and lexical access during word recognition in continuous speech. Cognitive Psychology, 10, 29-63.

McClelland, J. L., \& Elman, J. L. (1986). The TRACE model of speech perception. Cognitive Psychology, 18, 1-86.

MeYer, D. E., \& SChVANEVELdT, R. W. (1977). Facilitation in recognizing pairs of words: Evidence of a dependence between retrieval operations. Journal of Experimental Psychology, 90, 227-234.

MoRTon, J. (1969). Interaction of information in word recognition. Psychological Review, 76, 165-178.

NeELY, J. H. (1977). Semantic priming and retrieval from lexical memory: Roles of inhibitionless spreading activation and limited capacity attention. Journal of Experimental Psychology: General, 106, 226-254.

PosNer, M. I., \& SNYDER, C. R. (1975). Facilitation and inhibition in the processing of signals. In P. M. A. Rabbit \& S. Dornic (Eds.), Attention and performance $V$ (pp. 669-682). London: Academic Press.

RADEAU, M. (1983). Semantic priming between spoken words in adults and children. Canadian Journal of Psychology, 4, 547-556.

Scarborough, D. L., Cortese, C., \& Scarborough, H. S. (1977). Frequency and repetition effects in lexical memory. Journal of Experimental Psychology: Human Perception \& Performance, 3, 1-17.

Stevens, K. N., \& Blumstein, S. E. (1981). The search for invariant acoustic correlates of phonetic features. In P. D. Eimas \& J. L. Miller (Eds.), Perspectives on the study of speech (pp. 1-38). Hillsdale, NJ: Erlbaum.

TWeedy, J. R., Lapinski, R. H., \& SchVaneveldt, R. W. (1977). Semantic context effects on word recognition: Influence of varying the proportion of items presented in an appropriate context. Memory \& Cognition, 5, 84-89.

(Manuscript received for publication October 2, 1987.) 